\title{
ON THE INTRINSIC TOPOLOGY AND SOME RELATED IDEALS OF $C(X)$
}

\author{
O. A. S. KARAMZADEH AND M. ROSTAMI
}

\begin{abstract}
The above topology is defined and studied on $C(X)$, the ring of real-valued continuous functions on a completely regular Hausdorff space $X$. The minimal ideals and the socle of $C(X)$ are characterized via their corresponding $z$-filters. We observe that these ideals are $z$-ideals and $X$ is discrete if and only if the socle of $C(X)$ is a free ideal. It is also shown that for a class of topological spaces, containing all $P$-spaces, the family $C_{k}(X)$ of functions with compact support is identical with the intersection of the free maximal ideals of $C(X)$.
\end{abstract}

Introduction. Clearly every maximal ideal in any commutative ring with unity either intersects every nonzero ideal nontrivially or else is generated by an idempotent (in this case we call it isolated; see $[\mathbf{1 0}-\mathbf{1 3}]$ for more details). The existence of isolated maximal ideals of $C(X)$ is equivalent to the existence of isolated points in $X$, which plays a fundamental role in the study of some important topological spaces (see $[\mathbf{7}, \mathbf{2 2}, \mathbf{1 4}])$. In this paper we study the intrinsic topology on $C(X)$ and relate the density of the set of isolated points in $X$ to some algebraic properties of $C(X)$. The minimal ideals and the socle of $C(X)$, which is the sum of all the minimal ideals (see $[\mathbf{1}]$ ), are characterized via their corresponding $z$-filters. The equality of $C_{k}(X)$, the set of functions with compact support, with the intersection of the free maximal ideals was first proved for discrete spaces by Kaplansky [9], who asked if the equality holds in general. It is well known that the equality may fail in general and Kohls [15], Gillman-Jerison [4], and Robinson [20] have proved the result for $P$-spaces, realcompact spaces, and spaces admitting a complete uniform structure, respectively. We add a new class of topological spaces, larger than $P$-spaces, to these. The realcompactness of a discrete space with cardinality less than or equal to $c$ (the cardinality of the reals) is proved in a very simple way.

In this paper we denote by $X$ a completely regular Hausdorff space. If $f \in$ $C=C(X)$, then $Z(f)$ denotes the set of zeros of $f$. If $I$ is an ideal of $C$, then $Z[I]=\{Z(f): f \in I\}$ is a $z$-filter. The Stone-Cech compactification of $X$ is denoted by $\beta X$, and by $|X|$ we mean the cardinality of $X$. The reader is referred to [4] for undefined terms and notations.

1. Isolated points and isolated maximal ideals. We begin with the following well-known result.

Proposition 1.1. A point $x \in X$ is isolated if and only if $M_{x}=\{f \in$ $C: f(x)=0\}$ is an isolated maximal ideal of $C$.

Received by the editors March 29, 1983 and, in revised form, February 29, 1984.

1980 Mathematics Subject Classification. Primary 54C40.

Key words and phrases. Isolated maximal ideals, intrinsic topology, $P$-space, minimal ideal, socle, real pseudo-finite space, $P$-ideal, $z$-ideal. 
In the next result $R$ is a commutative semisimple ring with unity.

PROPOSITION 1.2. $M$ is an isolated maximal ideal of $R$ if and only if $M$ is an isolated point in $\operatorname{Max}(R)$, the space of maximal ideals of $R$ with the hull-kernel topology.

Proof. Let $M=e R$, where $e=e^{2}$, be an isolated maximal ideal of $R$. Then $\{M\}=\left\{M^{\prime} \in \operatorname{Max}(R): 1-e \notin M^{\prime}\right\}$. Conversely, if $\{M\}$ is open in $\operatorname{Max}(R)$, then $P=\bigcap\left\{M^{\prime} \in \operatorname{Max}(R): M^{\prime} \neq M\right\} \neq(0)$ implies $P \cap M=(0)$ and $P \oplus M=R$, i.e., $M$ is isolated.

REMARK 1.3. Using the above results, one can easily verify the well-known fact that every isolated point of $X$ is also isolated in $\beta X$.

2. The intrinsic topology. Let $Y$ be the set of isolated points of $X$, and for each finite subset $F$ of $Y$, let $C_{F}=\{f \in C: F \subseteq Z(f)\}$. Now take the ideals of the form $C_{F}$ as a base for the neighborhood system of the zero element in $C(X)$. Clearly $C$ with this topology is a topological ring (not necessarily Hausdorff). Since every $C_{F}$ is a finite intersection of isolated maximal ideals, this topology coincides with the intrinsic topology introduced by Goldman [6] for arbitrary rings. From now on we call it the In-topology on $C(X)$. We denote the intersection of the isolated maximal ideals by $J_{0}$. So $J_{0}=C$ if $Y=\emptyset$.

The next result relates the density of the isolated points in $X$ to some algebraic properties of $C(X)$.

PROPOSITION 2.1. For a topological space $X$, the following are equivalent:

1. The set of isolated points $Y$ of $X$ is dense in $X$.

2. The In-topology on $C$ is Hausdorff, i.e., $J_{0}=(0)$.

3. If $S$ is the socle of $C$, then $\operatorname{Ann}(S)=\{f \in C: S f=0\}=(0)$.

Proof. $(1) \rightarrow(2)$. Let $\bar{Y}=X$ and $f \in J_{0}$. Then clearly $f(Y)=0$ implies $f(\bar{Y}) \subset \overline{f(Y)}=0$, i.e., $f=0$.

$(2) \rightarrow(3)$. Clearly every minimal ideal of $C$ is generated by an idempotent, hence $S=\sum_{e \in E} \bigoplus e C$, where $E$ is a set of idempotents in $C$. We note that $e C$ is minimal if and only if $(1-e) C$ is an isolated maximal ideal, and $\operatorname{Ann}(e C)=(1-e) C$. But

$$
\operatorname{Ann}(S)=\bigcap_{e \in E} \operatorname{Ann}(e C)=\bigcap_{e \in E}(1-e) C=J_{0}=0 .
$$

$(3) \rightarrow(1)$. Clearly $\operatorname{Ann}(S)=0$ implies $J_{0}=(0)$. Now suppose $\bar{Y} \neq X$ and seek a contradiction. Let $x \in X-\bar{Y}$. Then there exists $f \in C$ such that $f(\bar{Y})=0$, $f(x) \neq 0$. But clearly $f(Y)=0$ implies $f \in J_{0}=(0)$, a contradiction.

EXAMPLES. If $X$ is either a countable Baire space (see $[\mathbf{7}, \mathrm{p} .16]$ ) or a scattered space (see $[\mathbf{2 2}, \mathbf{1 4}]$ ), then the In-topology on $C$ is Hausdorff. We note that if the In-topology on $C$ is Hausdorff, then $X$ is a Baire space (see $[\mathbf{7}$, p. 12]).

REMARK 2.2. Considering the isomorphism between $C^{*}(X)$ and $C(\beta X)$, one can easily see that $C(X)$ has a nontrivial In-topology if and only if $C^{*}(X)$ does. It is also obvious that the In-topology on $C(X)$ coincides with the relative topology for $C^{*}(X)$.

Clearly every open ideal in the In-topology on $C(X)$ is a finite intersection of isolated maximal ideals and is generated by an idempotent. In the next result we characterize closed ideals in this topology and note that our proof works for any commuative ring with unity. In what follows, $\bar{I}$ is the closure of $I$. 
Proposition 2.3. If $I$ is an ideal in $C(X)$, then either $\bar{I}=C$ or $\bar{I}$ is an intersection of isolated maximal ideals.

ProOF. We note that if $Y$, the set of isolated points of $X$ is empty, then $\bar{I}=C$. If $Y \neq \emptyset$, then $\bar{I}=\bigcap\left\{I+C_{F}: F \subseteq Y, F\right.$ is finite $\}$. But for each $F$, we have $C_{F}=$ $e_{1} C \cap e_{2} C \cap \cdots \cap e_{n} C$, where $e_{i} C, i=2, \ldots, n$, are isolated maximal ideals. Now if for each $C_{F}$ we have $I \nsubseteq e_{i} C, i=1,2, \ldots, n$, then $I+e_{i} C=C, i=1,2, \ldots n$. implies $I+C_{F}=C$ (see $[\mathbf{2 4}$, p. 177]) and, hence, $\bar{I}=C$. Therefore we assume that for some $C_{F}=e_{1} C \cap e_{2} C \cap \cdots \cap e_{n} C$, we have $I \subseteq e_{i} C$, for some $1 \leq i \leq n$. Put $F=F_{1} \cup F_{2}$, where $I \subseteq C_{F_{1}}$ and $I \nsubseteq C_{F_{2}}$. Now, by what we have already shown, we have $I+C_{F_{2}}=C$ and, since $I \subseteq C_{F_{1}}$, therefore, $I+C_{F_{2}} \cap C_{F_{1}}=C_{F_{1}}$. Thus $I+C_{F}=C_{F_{1}}$, which finishes the proof.

In what follows, by $C^{n}(X)$ we mean $C^{n}(X)=C\left(C^{n-1}(X)\right)$ and $C^{0}(X)=X$. Each $C^{n}(X)$ with its In-topology is considered as a topological space.

Proposition 2.4. The In-topology on $C^{n}(X), \forall n \geq 3$, is the trivial one and $C^{2}(X)$ has a nontrivial In-topology if and only if $X$ is finite.

ProOF. If $C^{n}(X)$ has a nontrivial In-topology for some $n \geq 3$, then $C^{n-1}(X)$ must have isolated points. This means that the zero ideal is open in the In-topology on $C^{n-1}(X)$, which shows that $C^{n-1}(X)$ has only a finite number of maximal ideals. Therefore $C^{n-2}(X)$ must be finite, which is impossible. Now if $C^{2}(X)$ has a nontrivial In-topology, then, by what we have just proved, $X$ must be finite. Conversely, if $X$ is finite, then clearly $C(X)$ is isomorphic with $R^{n}=R \times R \times \cdots \times R$ ( $n$ times), where $R$ is the reals. But the In-topology on $R^{n}$ is discrete and therefore the In-topology on $C^{2}(X)$ is nontrivial.

In [8] it is shown that the set of zero-divisors $D$ of $C(X)$ is the union of the minimal prime ideals. In case the set of isolated points is dense in $X$, we have the following, which shows that $D$ is open in the In-topology on $C(X)$.

PROPOSITION 2.5. If $Y$, the set of isolated points of $X$, is dense in $X$ then

$$
\begin{aligned}
D & =\{f \in C: Y \cap Z(f) \neq \emptyset\} \\
& =\bigcup\{M: M \text { is an isolated maximal ideal of } C(X)\} .
\end{aligned}
$$

Proof. Let $0 \neq f \in D$. Then there exists $0 \neq g \in C$ such that $f g=0$. Now there exists $y \in Y$ such that $g(y) \neq 0$. Hence $f(y) g(y)=0$ implies $y \in Z(f)$. Conversely, if $Z(f) \cap Y \neq \emptyset$, for some $f \in C$, then clearly $f$ is contained in an isolated maximal ideal. Thus $f$ is a zero-divisor. The second equality is obvious.

Next we give an easy proof of the fact that every discrete space of cardinality $\leq c$ is realcompact.

LEMMA 2.6. Suppose $A$ is the set of isolated points of $X$ and $|A| \leq c$. Now if $C(X)$ is isomorphic with $C(Y)$, where $Y$ is a discrete space, then $X$ is also discrete.

Proof. Since the In-topology on $C(X)$ is Hausdorff, $A$ is dense in $X$. Clearly $\beta X$ is homeomorphic with $\beta Y$. Hence $\beta X$ and $X$ are extremally disconnected. Since $|A| \leq c$, there exists a one-one function $f: A \rightarrow[0,1]$, which is clearly continuous on $A$. But since $X$ is extremally disconnected, $A$ is $C^{*}$-embedded (see $[4,6 \mathrm{M} .2]$ ). Therefore there exists $f^{\prime} \in C^{*}(X)$ such that $\left.f^{\prime}\right|_{A}=f$. Now we aim to show that every $P$-point of $X$ is isolated and, since $X$ is a $P$-space, we are through. To this 
end let $x \in X$ be a nonisolated $P$-point and seek a contradiction. By [4, 4L.3], $f^{\prime}$ is constant on a neighborhood $G$ of $x$. But $X$ is Hausdorff, so $G$ intersects $A$ in more than one point, a contradiction.

PROPOSITION 2.7. Every discrete space $X$ of cardinality $\leq c$ is realcompact.

ProOf. Consider the isomorphism between $C(X)$ and $C(v X)$, where $v X$ is the Hewitt realcompactification of $X$, and note that $X$ is the set of isolated points of $v X$. Now apply Lemma 2.6 .

The following is also immediate.

PROPOSITION 2.8. If $|X| \leq c$ and $C(X)$ is isomorphic with $C(Y)$, where $Y$ is a discrete space, then $X$ is also discrete.

3. Minimal ideals and the socle of $C(X)$. First we characterize the above ideals of $C(X)$ and then prove Theorem 3.8, our main result in this section.

Proposition 3.1. I is a nonzero minimal ideal of $C(X)$ if and only if $|Z[I]|=2$.

Proof. If $0 \neq I$ is a minimal ideal of $C(X)$, then $I=e C$, where $e$ is an idempotent such that $Z(e)=X-\{x\}$, for some $x \in X$. Hence $Z[I]=\{X, Z(e)\}$. Conversely if $|Z[1]|=2$, then $Z(f)=Z(g)$, for all nonzero elements $f, g$ in $I$. Now we claim that $Z(f)=X-\{x\}, \forall 0 \neq f \in I$ and some $x \in X$. Then $I=e C$, where $Z(e)=Z(f)$ and $e(x)=1$, which is clearly a minimal ideal. To prove our claim we show that $X-Z(f)$ cannot contain two distinct points. To see this let $x, y \in X-Z(f), x \neq y$. Then there exists $g \in C(X)$ such that $g(Z(f) \cup\{x\})=0$ and $g(y) \neq 0$. But $Z(f) \subsetneq Z(g)$ implies $Z(g) \in Z[I]$. Hence, we must have $Z(g)=X$, a contradiction.

\section{COROLlary 3.2. Every minimal ideal of $C(X)$ is a $z$-ideal.}

Proposition 3.3. The socle $S$ of $C(X)$ consists of all functions which vanish everywhere except on a finite number of points of $X$.

PROOF. We note that if $S=(0)$ then by considering the empty set as a finite set, the result is trivially true. Hence, suppose $S=\sum_{k \in K} \bigoplus I_{k}$, where $I_{k}$ runs over the set of minimal ideals of $C(X)$. Now for each $f \in S$ we have $f=f_{1}+f_{2}+\cdots+f_{n}$, where each $f_{i}$ belongs to some minimal ideal in $C(X)$. Then by Proposition 3.1 each $f_{i}$ is zero everywhere except at an isolated point $x_{i}$ of $X$. Thus $Z(f)=X-\left\{x_{1}, x_{2}, \ldots, x_{n}\right\}$. Conversely, let $X-Z(f)$ be a finite set; then we have to show that $f \in S$. But $X-Z(f)=\left\{x_{1}, x_{2}, \ldots, x_{n}\right\}$; then each $x_{k}, k=1,2, \ldots, n$, is an isolated point of $X$. Now for each $x_{k}$, there exists a minimal ideal $I_{k}$ such that $Z(f)=X-\left\{x_{k}\right\}$, for all nonzero $f \in I_{k}$. But each $I_{k}$ is of the form $I_{k}=e_{k} C$, where $e_{k}$ is an idempotent in $C$. Then, clearly, $f=e_{1} f+e_{2} f+\cdots+e_{n} f \in I_{1}+I_{2}+\cdots+I_{n} \subseteq S$.

\section{COROLlary 3.4. The socle of $C(X)$ is a $z$-ideal.}

REMARK 3.5 Using Proposition 2.3 or a result in [6], one can see that the socle $S$ of $C(X)$ and, therefore, $C_{k}(X) \supseteq S$ are dense in the In-topology on $C(X)$. 
COROLlaRY 3.6. $X$ is discrete if and only if the socle $S$ of $C(X)$ is a free ideal.

ProOF. We note that if $X$ is any topological space and $S \neq 0$, then $A=\bigcap Z[S]$ is the set of nonisolated points of $X$. The result is now obvious.

REMARK 3.7. Minimal ideals and the socle of $C(X)$ are $P$-ideals. (An ideal $I \neq 0$ is a $P$-ideal in $C(X)$ if every prime (proper) ideal of $I$ is maximal in $I$ (see [21, Lemma 1.2]).)

We observe that if $X$ is a pseudo-finite space (a space in which every compact subspace is finite), then the socle $S$ of $C(X)$ coincides with $C_{k}(X)$. It would be interesting to characterize topological spaces $X$ such that $S=C_{k}(X)$.

Next we generalize a result of Kohls $[\mathbf{1 5}]$ concerning the equality of $C_{k}(X)$ with the intersection of the free maximal ideals.

DEFINITION. A space $X$ is called real pseudo-finite if its Hewitt realcompactification $v X$ is pseudo-finite. Clearly every $P$-space is real pseudo-finite, but the converse is not true, for there are spaces $E$ and $V$ in $[\mathbf{2 3}]$ which are realcompact and pseudo-finite but not $P$-spaces.

For examples of pseudo-finite spaces which are not real pseudo-finite, see either [19, Example 2 or 3, p. 111].

THEOREM 3.8. If $X$ is a real pseudo-finite space, then $C_{k}(X)$ coincides with the intersection of the free maximal ideals of $C(X)$.

ProOF. Let $v X$ be the Hewitt realcompactification of $X$. Then by $[\mathbf{4}, 8.19]$, we have

$$
C_{k}(v X)=\bigcap\{M: M \in F \text {, where } F \text { is the set of free maximal ideals of } C(v X)\} \text {. }
$$

We note that $X$ is also pseudo-finite; therefore the socles of $C(X)$ and $C(v X)$ coincide with $C_{k}(X)$ and $C_{k}(v X)$, respectively. Now since the socle is stable under any isomorphism, (see also [16, p. 59]), then the isomorphism $\varphi: C(X) \rightarrow C(v X)$, where $\varphi(f)=f^{\prime}$, takes the socle of $C(X)$ onto the socle of $C(v X)$. Therefore,

$$
C_{k}(X)=\varphi^{-1}\left(C_{k}(v X)\right)=\bigcap\left\{\varphi^{-1}(M): M \in F\right\} .
$$

But clearly each $\varphi^{-1}(M)$ is a free maximal ideal in $C(X)$. This shows that $C_{k}(X)$ contains the intersection of the free maximal ideals. Hence we are through.

\section{REFERENCES}

1. F. F. Bonsall and J. Duncan, Complete normed algebras, Springer-Verlag, 1973.

2. J. G. Brookshear, On projective prime ideals in $C(X)$, Proc. Amer. Math. Soc. 69 (1978), 203-204.

3. W. W. Comfort, On the Hewitt realcompactification of a product space, Trans. Amer. Math. Soc. 131 (1968), 107-117.

4. L. Gillman and M. Jerison, Rings of continuous functions, Springer-Verlag, 1976.

5. L. Gillman, Countably generated ideals in rings of continuous functions, Proc. Amer. Math. Soc. 11 (1960), 660-666.

6. O. Goldman, A Wedderburn-Jacobson structure theorem, J. Algebra 34 (1975), 64-73.

7. R. C. Hayworth and R. A. McCoy, Baire spaces, Dissertationes Math. 141 (1977).

8. M. Henriksen and M. Jerison, The space of minimal prime ideals of a commutative ring, Trans. Amer. Math. Soc. 115 (1965), 110-130.

9. I. Kaplansky, Topological rings, Amer. J. Math. 69 (1947), 153-183. 
10. O. A. S. Karamzadeh, Projective maximal right ideals of self-injective rings, Proc. Amer. Math. Soc. 48 (1975), 286-288.

11. O. A. S. Karamzadeh and M. Motamedi, A note on rings in which every maximal ideal is generated by an idempotent, Proc. Japan Acad. Ser. A Math. Sci. 58 (1982), 124-125.

12. O. A. S. Karamzadeh, On maximal right ideals which are direct summands, Bull. Iran. Math. Soc. 18 (1983), 40-46.

13. O. A. S. Karamzadeh and M. Motamedi, On the intersection of maximal right ideals which are direct summands, Bull. Iran. Math. Soc. 18 (1983), 47-54.

14. O. A. S. Karamzadeh, On the classical Krull dimension of rings, Fund. Math. 117 (1983).

15. C. W. Kohls, Ideals in rings of continuous functions, Fund. Math. 45 (1975), 28-50.

16. J. Lambek, Rings and modules, Blaisdell, New York, 1966.

17. M. Mandelker, Supports of continuous functions, Trans. Amer. Math. Soc. 156 (1971), 73-83.

18. G. De Marco, On the countably generated $z$-ideals of $C(X)$, Proc. Amer. Math. Soc. 31 (1972), 574-576.

19. M. Rajagopalan and R. F. Wheeler, Sequential compactness of X implies a compactness property for $C(X)$, Canad J. Math. 28 (1976), 207-210.

$20 \mathrm{~S}$. M. Robinson, The intersection of the free maximal ideals in a complete space, Proc. Amer. Math. Soc. 17 (1966), 468-469.

21. D. Rudd, $P$-ideals and $F$-ideals in rings of continuous functions, Fund. Math. 88 (1970), 53-59.

22. W. Rudin, Continuous functions on compact spaces without perfect subsets, Proc. Amer. Math. Soc. 8 (1957), 39-42.

23. R. F. Wheeler, The strict topology for P-spaces, Proc. Amer. Math. Soc. 41 (1973), 466-467.

24. O. Zariski and P. Samuel, Commutative algebra, vol. 1, Van Nostrand, New York, 1958.

DePaRtment of MAThematics, JUNDi-Shapur UNiversity, AhVAZ, IRAN 